\title{
Gremlin 2 inhibits adipocyte differentiation through activation of $\mathrm{Wnt} / \boldsymbol{\beta}$-catenin signaling
}

\author{
QING WU ${ }^{1}$, SHI-GUO TANG ${ }^{2}$ and ZHONG-MING YUAN ${ }^{3}$ \\ ${ }^{1}$ Department of Geriatrics Cardiology, The Second Affiliated Hospital of Chongqing Medical University; \\ ${ }^{2}$ Department of Endocrinology, Chongqing Ninth People's Hospital; ${ }^{3}$ Department of Geriatrics, \\ The Second Affiliated Hospital of Chongqing Medical University, Chongqing 400010, P.R. China
}

Received February 13, 2014; Accepted October 31, 2014

DOI: $10.3892 / \mathrm{mmr} .2015 .4117$

\begin{abstract}
The primary function of white adipose tissues is to store excess energy. The current study aimed to investigate the roles of Gremlin 2 (Grem2), a glycoprotein in adipogenesis. Using polymerase chain reaction-based microarrays, it was determined that Grem 2 was markedly downregulated in adipose tissues from obese animals and humans. In addition, 3T3-L1 cells were used to investigate the details of the mechanisms underlying the anti-adipogenic effects of Grem2. Grem 2 expression was markedly decreased upon the induction of adipocyte differentiation, as demonstrated by reverse transcription-quantitative polymerase chain reaction and western blot analysis. Notably, Grem 2 overexpression inhibited adipogenesis, while knockdown of Grem 2 led to an increase in adipogenesis. At the molecular level, Grem 2 promotes nuclear translocation of $\beta$-catenin, an integral Wnt signaling component. Consistently, inhibition of $\mathrm{Wnt} / \beta$-catenin signaling using a retrovirus targeting the $\beta$-catenin coding region attenuated the anti-adipogenic effects of Grem2. Therefore, to the best of our knowledge, the current study shows for the first time that Grem 2 may be an important regulator of adipocyte differentiation.
\end{abstract}

\section{Introduction}

When dietary nutrient intake chronically exceeds energy expenditure, obesity occurs due to an increase in the size and/or number of white adipocytes (1). Obesity is closely associated with type 2 diabetes, fatty liver, hyperlipidemia, cardiovascular diseases and tumorigenesis $(2,3)$. Thus, investigating the molecular and cellular mechanisms regulating adipocyte

Correspondence to: Dr Zhong-Ming Yuan, Department of Geriatrics, The Second Affiliated Hospital of Chongqing Medical University, 76 Linjiang Road, Chongqing 400010, P.R. China

E-mail: yuan_zhongming@126.com

Key words: adipogenesis, Gremlin2, Wnt $/ \beta$-catenin signaling, adipocyte differentiation differentiation is crucial for designing potential therapies to prevent obesity and the associated diseases.

Decades of studies have identified a number of transcription factors involved in adipocyte differentiation, including peroxisome proliferator-activated receptor $\gamma(\operatorname{PPAR} \gamma)$ and members of the $\mathrm{C} / \mathrm{EBP}$ family $(4,5)$. Additionally, a number of other factors or signaling molecules are involved in the control of adipocyte differentiation, such as Wnt/ $\beta$-catenin, cAMP/CREB, and the family of kruppel-like factor (KLF) proteins (6-8). Furthermore, recent studies have indicated that modification of these proteins has an important role in adipogenesis. It has been shown that phosphorylation, acetylation and small ubiquitin-like modifier (SUMO)ylation of PPAR $\gamma$ affects adipocyte differentiation, though regulation of its transcriptional activity (9-11). In this regard, the process of adipogenesis is rather complex, suggesting that identification of the additional factors may provide novel insights into the mechanisms regulating the initiation of fat cell differentiation and obesity.

In the present study, polymerase chain reaction (PCR)-based microarrays were performed in obese and lean mice to screen potential genes involved in adipogenesis.

\section{Materials and methods}

Mice. Male C57BL/6, ob/ob and $d b / d b$ mice, aged 10-12 weeks, were purchased from the Shanghai Laboratory Animal Company (Shanghai, China). All mice were housed and bred according to international standard conditions, with a 12-h dark/light cycle. For induction of obesity, mice aged 10 weeks were placed on a high-fat-diet (50 kcal\% fat, $25 \mathrm{kcal} \%$ protein and $25 \mathrm{kcal} \%$ carbohydrate) for 12 weeks. The normal diet consisted of $10 \mathrm{kcal} \%$ fat, $15 \mathrm{kcal} \%$ protein and $75 \mathrm{kcal} \%$ carbohydrate. The animal protocol was reviewed and approved by the Animal Care Committee of Chongqing Ninth People's Hospital (Chongqing, China).

Human tissue samples. Human subcutaneous fat tissues from obese $(\mathrm{BMI}>25 ; \mathrm{n}=25)$ and lean $(\mathrm{BMI}<23 ; \mathrm{n}=20)$ adults were collected at the Department of Endocrinology at Chongqing Ninth People's Hospital. All subjects had a stable weight, defined as the absence of fluctuations of $>1 \%$ of body weight for at least 2 months prior to surgery. Adipose tissue was 
immediately frozen in liquid nitrogen following extraction. All samples were obtained with informed consent and the study was approved by the Chongqing Ninth People's Hospital Institutional Review Board.

Cell culture. 3T3-L1 fibroblasts were obtained from The Cell Bank of Type Culture Collection of Chinese Academy of Sciences (Shanghai, China), and cultured in Dulbecco's modified Eagle's medium (DMEM; Gibco-BRL, Carlsbad, CA, USA) supplemented with $10 \%$ fetal bovine serum (FBS; Gibco-BRL), $100 \mathrm{IU} / \mathrm{ml}$ penicillin and $100 \mu \mathrm{g} / \mathrm{ml}$ streptomycin (Gibco-BRL). Adipocyte differentiation was induced by treating the cells at $80-90 \%$ confluency with standard differentiation inducers [100 $\mu \mathrm{M}$ IBMX (Beyotime Institute of Biotechnology, Nantong, China), $100 \mathrm{nM}$ dexamethasone (Beyotime Institute of Biotechnology) and $10 \mu \mathrm{g} / \mathrm{ml}$ insulin (Eli Lilly \& Co., Indianapolis, IN, USA)] for $48 \mathrm{~h}$ (from day 0 to day 2). Cells were treated with DMEM supplemented with $10 \mu \mathrm{g} / \mathrm{ml}$ insulin and 10\% FBS for another $48 \mathrm{~h}$ (from day 2 to day 4). Subsequently, the medium was replaced by DMEM and $10 \%$ FBS, which was changed daily, and the cells were cultured for a further 4-6 days.

RNA extraction, $c D N A$ microarrays, and reverse transcription-quantitative polymerase chain reaction (RT-qPCR). Total RNA was isolated from tissues or cells using the TRIzol reagent (Invitrogen Life Technologies, Shanghai, China), and reverse transcription was performed using the RT kit from Promega Corporation (Madison, WI, USA), according to the manufacturer's instructions. Affymetrix arrays were performed using Mouse Genome 2.0 chips from Gene Tech Company Limited (Shanghai, China). In order to quantify the transcripts of the interest genes, qPCR was performed using a SYBR Green Premix Ex Taq kit (Takara Bio Inc., Dalian, China) on a Light Cycler 480 (Roche, Basel, Switzerland) with $5 \mathrm{mg}$ RNA. The following primer sequences form Bioyare Biotechnology Company (Shanghai, China) were used: Mouse Grem2, forward 5'-TGTGCTGGT AAAGGTAGCTGA-3' and reverse 5'-CCACCTCTCTGA GTTGTTGCT-3'; human Grem2, forward 5'-ATCCCCTCG CCTTACAAGGA-3' and reverse 5'-TCTTGCACCAGT CACTCTTGA-3'; C/EBPa, forward 5'-CAAGAACAGCAA CGAGTACCG-3' and reverse 5'-GTCACTGGTCAACTC CAGCAC-3'; PPARg, forward 5'-CTCCAAGAATACCAA AGTGCGA-3' and reverse 5'-GCCTGATGCTTTATCCCC ACA-3'; LPL, forward 5'-ATGGATGGACGGTAACGG GAA-3' and reverse 5'-CCCGATACAACCAGTCTACTA CA-3'; PEPCK, forward 5'-ATGAGCCGCAAACTGGGT C-3' and reverse 5'-AGAGCCGAACTCCACAATCTC-3'; CD36, forward 5'-AGCATTCAACGCCAGGTTC-3' and reverse 5'-CGAGTCTGTCAGTTCAATACCAA-3'; aP2, forward 5'-AAGGTGAAGAGCATCATAACCCT-3' and reverse 5'-TCACGCCTTTCATAACACATTCC-3'; mouse HPRT1, forward 5'-TCAGTCAACGGGGGACATAAA-3' and reverse 5'-GGGGCTGTACTGCTTAACCAG-3'; human HPRT1, forward 5'-CCTGGCGTCGTGATTAGTGAT-3' and reverse 5'-AGACGTTCAGTCCTGTCCATAA-3'. The PCR cycling conditions were as follows: Initial period at $94^{\circ} \mathrm{C}$ for 3 min, followed by a two-step PCR program consisting of $95^{\circ} \mathrm{C}$ for $5 \mathrm{sec}$ and $60^{\circ} \mathrm{C}$ for $30 \mathrm{sec}$ for 55 cycles. Gene expression was calculated relative to the mRNA expression of HPRT1, and relative quantitation analysis of gene expression data was performed using the $2^{-\Delta \Delta \mathrm{Ct}}$ method.

Retrovirus, small interfering (si)RNA and transfection. Retroviruses containing empty vector or Grem 2 cDNA were constructed by Invitrogen Life Technologies. siRNA oligos targeting Grem2 or $\beta$-catenin were purchased from Gene Pharma (Shanghai, China) and transfected into 3T3-L1 cells using Lipofectamine 2000 (Invitrogen Life Technologies), according to the manufacturer's instructions. Histological analysis was conducted using Oil Red O staining. Cells were fixed with $4 \%$ paraformaldehyde for $30 \mathrm{~min}$, followed by incubation with Oil Red O (Sigma-Aldrich, St. Louis, MO, USA) for $45 \mathrm{~min}$.

Western blot analysis. Cells or tissues were harvested and lysed with lysis buffer $(150 \mathrm{mM} \mathrm{NaCl}, 50 \mathrm{mM}$ Tris- $\mathrm{HCl}$ and $1 \%$ NP-40; pH 7.5). Proteins were quantified and separated by $10 \%$ SDS-PAGE and transferred to polyvinylidine difluoride membranes (Amersham Bioscience, Buckinghamshire, UK). After blocking with $10 \%$ non-fat milk in phosphate-buffered saline, membranes were immunoblotted with the following rabbit antibodies from Abcam (Cambridge, MA, USA): Polyclonal anti-Grem2 (ab102563; 1:1,000), monoclonal C/EBP $\alpha$ (ab40764; 1:1,000), polyclonal PPAR $\gamma$ (ab19481; 1:1,000), monoclonal P-P38 (ab178867; 1:2,000), polyclonal P-AKT (ab66138; 1:1,000), monoclonal P-CREB (ab32096; 1:2,000), monoclonal CREB (ab32515; 1:2,000), polyclonal $\beta$-catenin (ab6302; 1:1,000), polyclonal lamin B (ab16048; $1: 2,000)$ and monoclonal GAPDH (ab181602; $1: 1,000)$. The blots were then incubated with horseradish peroxidase-linked secondary antibodies (Cell Signaling Technology, Inc. Danvers, MA, USA). The signals were detected using the SuperSignal West Pico Chemiluminescent Substrate kit (Pierce, Rockford, IL, USA) according to manufacturer's instructions.

Statistical analysis. The results are expressed as the mean \pm standard error of the mean and analyzed using Student's t-test or one way analysis of variance with GraphPad Prism software, version 5.0.1 (GraphPad Software, Inc., La Jolla, CA, USA). $\mathrm{P}<0.05$ was considered to indicate a statistically significant difference.

\section{Results}

Downregulation of Grem 2 in obese mice and humans. To identify potential genes involved in adipogenesis and obesity, male C57BL/6 mice aged 10 weeks were placed on high-fat or normal diets for 12 weeks. The mice were sacrificed by cervical dislocation and gene expression arrays were performed using epididymal adipose tissues. The clustering analysis of the Affymetrix arrays revealed that 1,174 genes were upregulated and 1,572 genes were downregulated in the adipose tissues of mice fed a high-fat-diet compared with those fed a normal diet $(\mathrm{P}<0.05$; data not shown). Among these genes, it was determined that Grem2 was significantly downregulated $(\mathrm{P}<0.001)$. Its downregulation was further confirmed by RT-qPCR and western blot analysis (Fig. 1A and B). 
A

Grem2

B
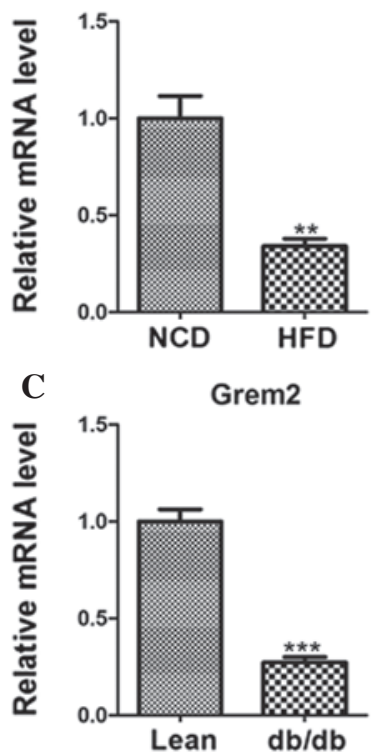

D

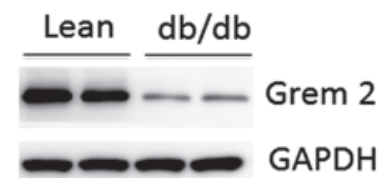

$\mathbf{F}$
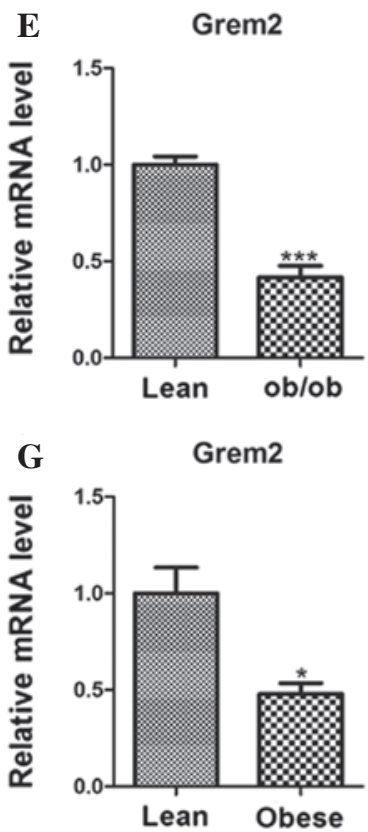

Figure 1. Downregulation of Gremlin 2 (Grem2) in adipose tissues from obese mice and humans. (A-F) mRNA and protein levels of Grem2 were determined in epididymal adipose tissues from obese mice. C57BL/6 mice were fed a high-fat-diet (HFD) or normal chow diet (NCD) for 12 weeks. After fasting for $8 \mathrm{~h}$, mice were sacrificed and tissues were collected. $\mathrm{n}=5-8$. ${ }^{*} \mathrm{P}<0.05,{ }^{* *} \mathrm{P}<0.01,{ }^{* * *} \mathrm{P}<0.001$. (G and $\left.\mathrm{H}\right)$ mRNA and protein levels of Grem2 were determined in subcutaneous adipose tissues from obese and lean human subjects.

To determine whether the inhibition of Grem 2 represents a common feature of obesity, $d b / d b$ and $o b / o b$ mice were employed as models for monogenic-induced obesity. Indeed, Grem2 mRNA and protein levels were found to be reduced in the epididymal adipose tissues of these mice, as compared with lean controls (Fig. 1C-F). Notably, Grem2 mRNA and protein levels were significantly reduced in subcutaneous fat from obese humans (Fig. 1G and H), further suggesting that the downregulation of Grem 2 expression may be a conserved feature in obese mice and humans.
A

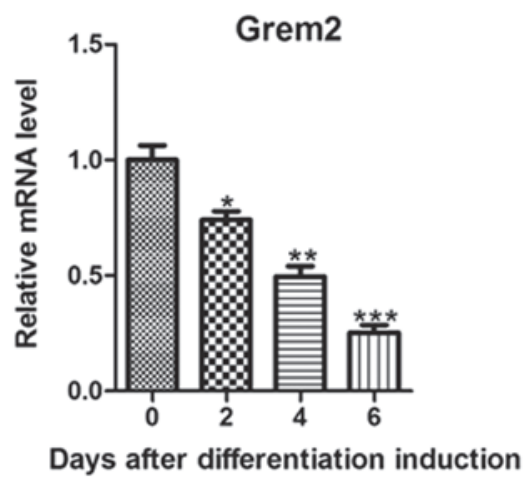

B

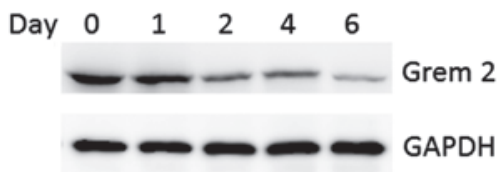

Figure 2. Gremlin 2 (Grem2) expression during 3T3-L1 adipocyte differentiation. Expression of Grem 2 mRNA and protein during differentiation of 3T3-L1 cells was analyzed using (A) reverse transcription-quantitative polymerase chain reaction and (B) western blot analysis at the indicated times ${ }^{*} \mathrm{P}<0.05,{ }^{* *} \mathrm{P}<0.01,{ }^{* * *} \mathrm{P}<0.001$ compared with day 0 .

Grem2 suppresses the differentiation of 3T3-L1 preadipocytes into adipocytes. To explore the functions of Grem2 in adipogenesis, its gene expression was investigated at numerous time points during 3T3-L1 differentiation. The RT-qPCR results revealed that Grem 2 mRNA gradually reduced from day 0 post-induction (Fig. 2A). The protein levels for Grem2 were consistent with its mRNA levels (Fig. 2B).

The role of Grem 2 in adipogenesis was tested using retroviruses carrying Grem 2 cDNA or empty vector, which resulted in forced overexpression of Grem2 in 3T3-L1 preadipocytes (Fig. 3A). These cells were cultured to confluence and differentiated using the standard induction cocktail for 8 days. Cell morphology indicated that cells overexpressing Grem2 accumulated significantly less lipids than the control cells (Fig. 3B). In addition, the expression levels of adipogenic regulators such as C/EBP $\alpha$ and PPAR $\gamma$ were reduced (Fig. 3C and D), which was consistent with the Oil Red O staining. Furthermore, markers of mature adipocytes LPL, PEPCK, CD36 and aP2, which are downstream targets of C/ EBP $\alpha$ and PPAR $\gamma$, were repressed by Grem 2 overexpression (Fig. 3E).

Grem 2 knockdown promotes adipocyte differentiation. To further examine the roles of Grem2, its specific siRNA was transfected into 3T3-L1 cells (Fig. 4A). As a result, knockdown of Grem2 levels showed a clear increase in the adipogenic potential of the 3T3-L1 cells compared with that of the cells treated with the negative control siRNA, as illustrated by cell morphology (Fig. 4B). Furthermore, knockdown of Grem2 increased expression levels of the adipocyte markers including C/EBP $\alpha$, PPAR $\gamma$, LPL, PEPCK, CD36 and aP2 (Fig. $4 \mathrm{C}$ and D). Together, these results indicate that Grem2 is a critical negative regulator in the adipogenesis. 
A

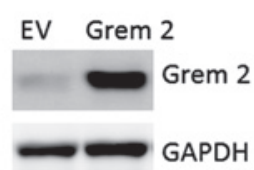

B

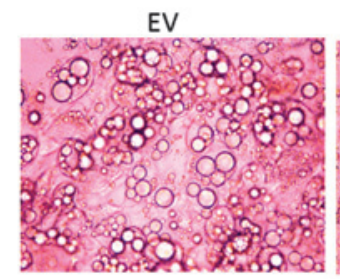

Grem 2

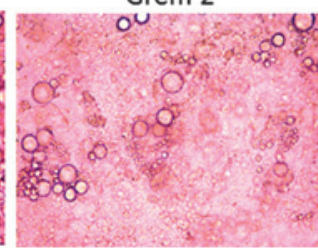

C

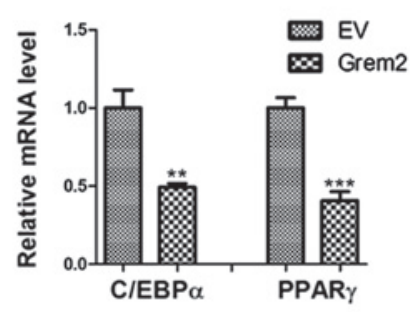

$\mathbf{E}$
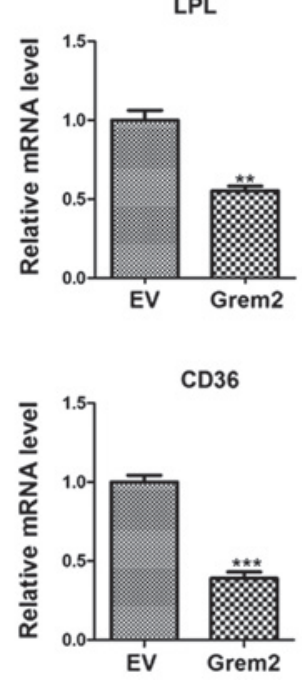

D

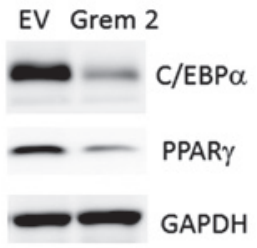

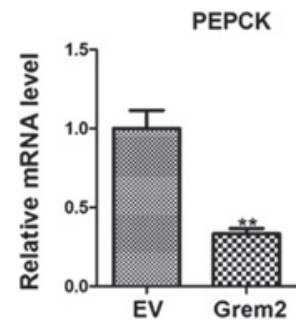

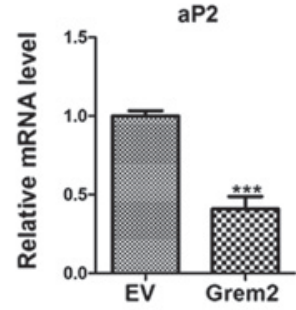

Figure 3. Gremlin 2 (Grem2) overexpression inhibits differentiation of 3T3-L1 preadipocytes into adipocytes. (A) Grem2 protein levels in 3T3-L1 cells after transfection with retrovirus containing empty vector (EV) or Grem2 cDNA. (B) 3T3-L1 cells were infected with retrovirus containing empty vector (EV) or Grem 2 cDNA. On day 8 after induction of differentiation, cell morphology was determined with Oil Red $\mathrm{O}$ staining and a microscope at $\mathrm{x} 200$. (C and D) mRNA and protein levels of C/EBP $\alpha$ and PPAR $\gamma$ in 3T3-L1 cells on day 8 after induction of differentiation. (E) mRNA levels of LPL, PEPCK, CD36 and aP2 in 3T3-L1 cells ${ }^{* *} \mathrm{P}<0.01,{ }^{* * *} \mathrm{P}<0.001$, compared with $\mathrm{EV}$.

Grem 2 activates Wnt/ $\beta$-catenin signaling in adipocytes. Finally, the molecular basis for the observed phenotypic changes in 3T3-L1 cells with Grem2 overexpression was investigated. As shown in Fig. 5A, Wnt signaling was highly activated by Grem 2 overexpression, as evidenced by nuclear accumulation of $\beta$-catenin, while other signaling molecules, including p38, AKT and cAMP/CREB, remained unaffected (Fig. 5B). Subsequently, it was investigated whether ablation of $\beta$-catenin reversed the roles of Grem2. A retrovirus targeting a $\beta$-catenin coding region (sh-catenin) was generated using pAD_BLOCK_IT_DEST vectors (Invitrogen Life Technologies, Grand Island, NY, USA) to knockdown endogenous $\beta$-catenin expression in 3T3-L1 cells (Fig. 5C). As a result, sh-catenin largely attenuated the inhibitory roles of Grem 2 on the adipogenesis, as evidenced by gene expression of adipogenic markers (Fig. 5D), suggesting that the role of Grem 2 in the adipogenesis, at least in part, relies on Wnt/ $\beta$-catenin activation.

\section{Discussion}

In the present study, it was determined that the Grem 2 gene was significantly downregulated in white adipose tissue from obese mice and humans. Using 3T3-L1 cells cultured in vitro, it was demonstrated that Grem 2 inhibited adipocyte differentiation. Thus, for the first time to the best of our knowledge, these results identify Grem 2 as a key regulator in adipose formation and obesity.

Members of the Gremlin family, including Grem2, have been shown to be bone morphogenetic protein (BMP) antagonists (12). Indeed, Grem2 regulates BMP signaling in cardiomyocyte and osteoblast differentiation $(13,14)$. Furthermore, a recent human study revealed that genetic variants in Grem 2 are associated with bone mineral density (15). Since certain members of the family of BMPs support white and brown adipocyte differentiation (16,17), whether Grem2 regulates BMP-induced adipogenesis requires further investigation. 
A

Day 2

siRNA

NC Grem2

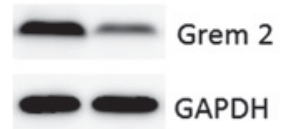

B

siRNA

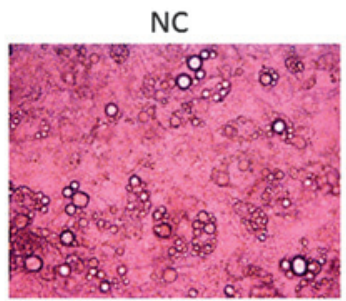

C

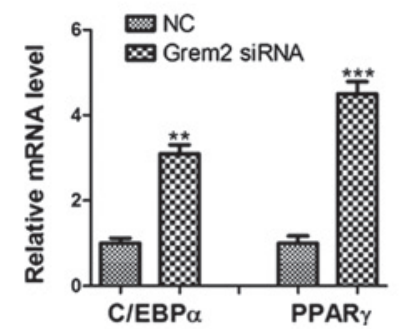

D
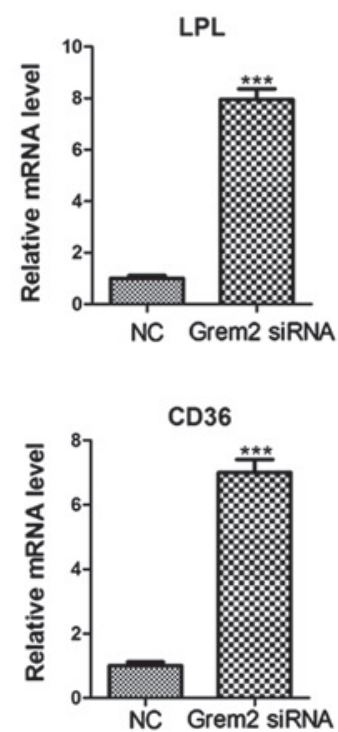

Day 4

siRNA NC Grem2
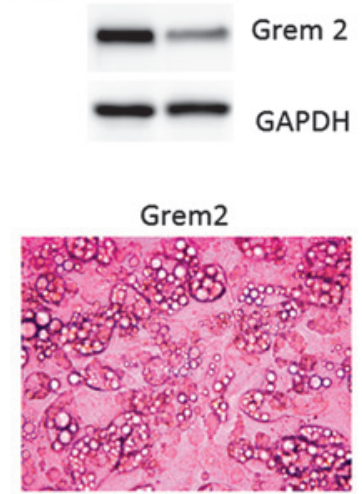

siRNA NC Grem2
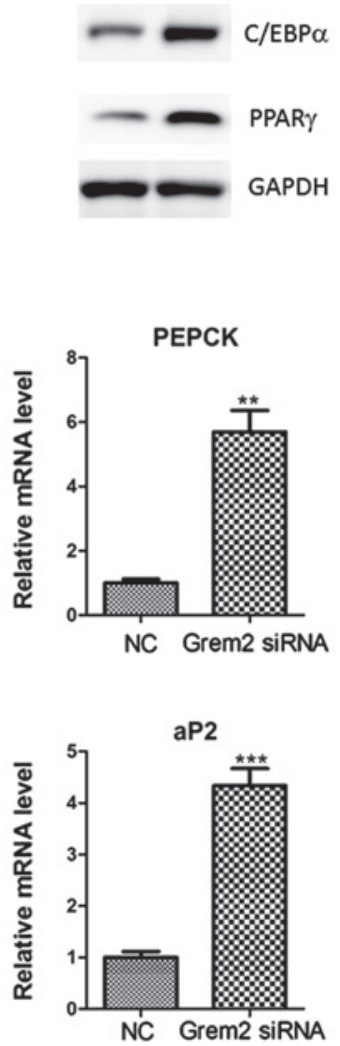

Figure 4. Gremlin 2(Grem2) knockdown promotes 3T3-L1 cell differentiation. (A) Grem 2 protein levels in 3T3-L1 cells after transfection with small interfering (si)RNA oligos targeting Grem 2 or negative controls. Cells were harvested at day 2 and day 4 after differentiation induction for western blot analysis. (B) 3T3-L1 cells were infected with siRNA oligos targeting Grem2 or negative controls. On day 8 after induction of differentiation, cell morphology was determined with Oil Red O staining and a microscope at x200. (C and D) Expression levels of C/EBP $\alpha$, PPAR $\gamma$, LPL, PEPCK, CD36 and aP2 in 3T3-L1 cells ${ }^{* *} \mathrm{P}<0.01,{ }^{* * *} \mathrm{P}<0.001$, compared with $\mathrm{NC}$.

At the molecular level, it was revealed that Grem2 activates $W n t / \beta$-catenin signaling, as evidenced by its nuclear accumulation. Initial studies have demonstrated that ectopic expression of Wnt1, an activator of Wnt/ $\beta$-catenin signaling, may inhibit adipogenesis $(6,18,19)$. Concurrently, pharmacological compounds that activate Wnt/ $\beta$-catenin signaling or
A

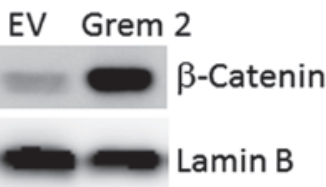

C

Day 2

NC sh-Catenin
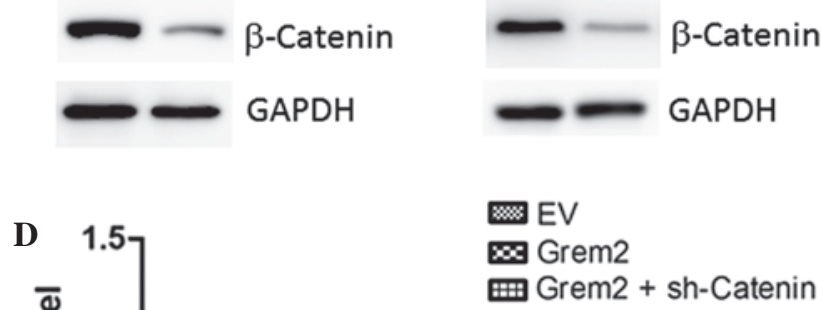

B

EV Grem 2

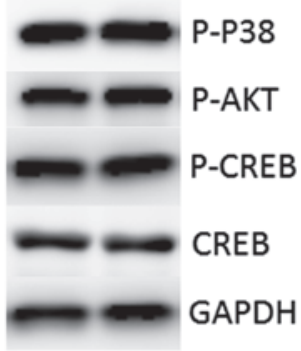

Day 4

NC sh-Catenin

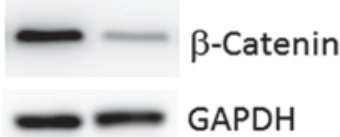

표 Grem2 + sh-Catenin

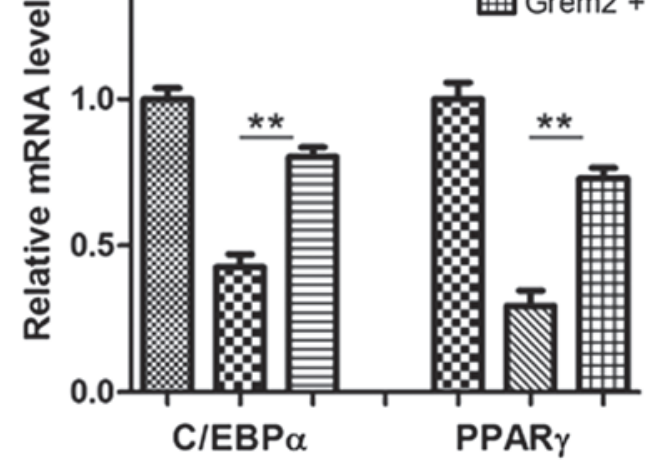

Figure 5. Gremlin 2 (Grem2) activates Wnt/ $\beta$-catenin signaling to inhibit adipogenesis. (A) Protein levels of nuclear $\beta$-catenin was determined by western blot analysis in 3T3-L1 cells after transfection of Grem2 or empty vector (EV). (B) Protein levels of phosphorylated p38, AKT and CREB were examined by western blot analysis in 3T3-L1 cells after transfection of Grem2 or EV. (C) Grem2 protein levels in 3T3-L1 cells after transfection with siRNA oligos targeting $\beta$-catenin or negative controls. Cells were harvested at days 2 and 4 after differentiation induction for western blot analysis. (D) Expression levels of $\mathrm{C} / \mathrm{EBP} \alpha$ and PPAR $\gamma$ in 3T3-L1 cells. ${ }^{* *} \mathrm{P}<0.01$.

transfection of stable $\beta$-catenin block preadipocyte differentiation (18). However, inhibition of Wnt signaling promotes cell differentiation $(18,20)$, indicating that Wnt signaling has a negative role in the adipocyte differentiation. In accordance with this conclusion in vitro, transgenic mice overexpressing $W n t 10 b$, an endogenous factor for Wnt signaling, have a $50 \%$ reduction in adiposity under a normal chow diet (21). These mice resist expansion of adipose tissue under conditions of high-fat-diet-induced and genetic-induced obesity (21). Furthermore, recent genetic research indicated the importance of Wnt $/ \beta$-catenin signaling in human obesity. Polymorphisms of the Wnt10b gene may be associated with obesity in human subjects of European origin (22). Together, these data suggest that modulators of $\mathrm{Wnt} / \beta$-catenin pathway may represent a useful therapeutic target for obesity (23).

In conclusion, the results of the current study highlight a pivotal role for Grem 2 in white fat-cell differentiation by controlling Wnt/ $\beta$-catenin signaling. Further studies are 
required to investigate the functions and mechanisms of Grem2 in vivo, using knockout or transgenic animals.

\section{References}

1. Rueda-Clausen CF, Padwal RS and Sharma AM: New pharmacological approaches for obesity management. Nat Rev Endocrinol 9: 467-478, 2013.

2. Fenske W, Athanasiou T, Harling L, Drechsler C, Darzi A and Ashafian H: Obesity-related cardiorenal disease: the benefits of bariatric surgery. Nat Rev Nephol 9: 539-551, 2013.

3. Tao W and Lagergren J: Clinical management of obese patients with cancer. Nat Rev Clin Oncol 10: 519-533, 2013.

4. Rosen ED and MacDougald OA: Adipocyte differentiation from the inside out. Nat Rev Mol Cell Biol 7: 885-896, 2006.

5. Kawai M and Rosen CJ: PPARy: A circadian transcription factor in adipogenesis and osteogenesis. Nat Rev Endocrinol 6 : 629-636, 2010

6. Cristancho AG and Lazar MA: Forming functional fat: a growing understanding of adipocyte differentiation. Nat Rev Mol Cell Biol 12: 722-734, 2011.

7. Oishi Y, Manabe I, Tobe K, et al: Kruppel-like transcription factor klf5 is a key regulator of adipocyte differentiation. Cell Metab 1: 27-39, 2005.

8. Birsoy K, Chen Z and Friedman J: Transcriptional regulation of adipogenesis by klf4. Cell Metab 7: 339-347, 2008.

9. Choi JH, Banks AS, Estall JL, et al: Anti-diabetic drugs inhibit obesity-linked phosphorylation of PPARgamma by cdk5. Nature 466: 451-456, 2010.

10. Qiang L, Wang L, Kon N, et al: Brown remodeling of white adipose tissue by sirt1-dependent deacetylation of ppargamma. Cell 150: 620-632, 2012.

11. Dutchak PA, Katafuchi T, Bookout AL, et al: Fibroblast growth factor-21 regulates PPARgamma activity and the antidiabetic actions of thiazolidinediones. Cell 148: 556-567, 2012.

12. Zuniga E, Rippen M, Alexander C, Schilling TF and Crump JG: Gremlin 2 regulates distinct roles of bmp and endothelin 1 signaling in dorsoventral patterning of the facial skeleton. Development 138: 5147-5156, 2011.
13. Suzuki D, Yamada A, Aizawa R, et al: bmp2 differentially regulates the expression of gremlin1 and gremlin2, the negative regulators of bmp function, during osteoblast differentiation. Calcif Tissue Int 91: 88-96, 2012.

14. Muller II, Melville DB, Tanwar V, et al: Functional modeling in zebrafish demonstrates that the atrial-fibrillation-associated gene GREM2 regulates cardiac laterality, cardiomyocyte differentiation and atrial rhythm. Dis Models Mech 6: 332-341, 2013.

15. Paternoster L, Lorentzon M, Lehtimaki T, et al: Genetic determinants of trabecular and cortical volumetric bone mineral densities and bone microstructure. PLoS Genet 9: e1003247, 2013.

16. Macotela Y, Emanuelli B, Mori MA, et al: Intrinsic differences in adipocyte precursor cells from different white fat depots. Diabetes 61: 1691-1699, 2012.

17. Takahashi A, Morita M, Yokoyama K, Suzuki T and Yamamoto T: Tob2 inhibits peroxisome proliferator-activated receptor gamma2 expression by sequestering smads and c/ebpalpha during adipocyte differentiation. Mol Cell Biol 32: 5067-5077, 2012.

18. Ross SE, Hemati N, Longo KA, et al: Inhibition of adipogenesis by wnt signaling. Science 289: 950-953, 2000.

19. Laudes M: Role of WNT signalling in the determination of human mesenchymal stem cells into preadipocytes. J Mol Endocrinol 46: R65-R72, 2011.

20. Prestwich TC and Macdougald OA: Wnt/beta-catenin signaling in adipogenesis and metabolism. Curr Opin Cell Biol 19: 612-617, 2007.

21. Wright WS, Longo KA, Dolinsky VW, et al: WNT10b inhibits obesity in ob/ob and agouti mice. Diabetes 56: 295-303, 2007.

22. Chistodoulides C, Scarda A, Granzotto M, et al: WNT10B mutations in human obesity. Diabetologia 49: 678-684, 2006.

23. Takada I, Kouzmenko AP and Kato S: Wnt and PPARgamma signaling in osteoblastogenesis and adipogenesis. Nat Rev Rheumatol 5: 442-447, 2009. 